\title{
8.3-4
}

\section{On the Willingness to Pay in Relation to Delivered Quality of Mobile Video Streaming}

\author{
Michal Ries, Olivia Nemethova and Markus Rupp \\ Institute of Communications and Radio-Frequency Engineering, Vienna University of Technology \\ Gusshausstasse 25, A-1040 Vienna, Austria \\ email: (mries, onemeth, mrupp)@nt.tuwien.ac.at \\ web: www.nt.tuwien.ac.at
}

\begin{abstract}
Provisioning of mobile video streaming became reality. The next evolution step in mobile video streaming is to allocate resources optimally from both the service provider and the user perspective. Therefore, it is important to determine the relation between the willingness to pay (WTP) and the delivered quality of mobile video streaming. In order to determine this relation we performed tests on subjective quality and WTP with the most frequent content types, codecs and their settings. A further analysis shows clearly a mutual dependence between subjective video quality and WTP.
\end{abstract}

\section{INTRODUCTION}

Provisioning of mobile multimedia services in suitable quality is gaining importance due to market and terminal development in recent years. The most challenging quality issue for provisioning of multimedia services is providing video streaming services in a required level of customer satisfaction. This required level of customer satisfaction is achieved with suitable combinations of codec and network settings for streamed content in order to allocate the resources optimally from both the service provider and the user perspective.

The multimedia mobile terminals have different size, mobility features and usage. This variety of terminals brings in new usage scenarios for multimedia services. Moreover, the usage scenario influences the human perception [1] significantly. Test scenarios on the other hand should consider real usage scenarios, in order to avoid systematic test failures.

Mobile video streaming is characterized by low resolutions and low bit rates. Currently, the codec mandatory for UMTS video streaming is H.263. Video streams encoded with H.263 contain a lot of video coding artifacts due to high compression ratios common in mobile streaming. This results in a significant video quality reduction. Nevertheless, H.263 is widely used because it is license-free. UMTS optional codecs are MPEG4 and since release 6 [2] a baseline profile of the H.264/AVC codec [3] is supported. The most suitable video coding standard for mobile streaming is H.264/AVC. Thanks to its significant improvement in video compression gain the newest video coding standard H.264/AVC allows to provide video streaming for low bit and frame rates while preserving perceptual quality.

Technically WTP has emerged from Subjective or Perceived/Perceptual QoS (PQ) [4] and is solely based on human perception or satisfaction regarding service usability. Determining PQ is typically carried out by surveying a set of persons, which participate in a controlled experiment [5]. Furthermore, the human visual perception of multimedia content is determined by the character of the observed sequence [6], [7]. The sequence character reflects motion characteristics (content type, video motion features, spatial information) [6], [7]. The recent trends show that the perceptual video QoS is defined by a set of intrinsic QoS parameters [4] as well as audio and video parameters.

Service providers are still trying to introduce new billing strategies in order to stimulate their consumers. The actual proposals for mobile streaming are focusing on particular contents or settings, not allowing such results to be generalized. There have been already complex QoS studies regarding WTP [8] and proposals of WTP utility functions for Internet streaming [9]. Unfortunately, these results are not applicable for mobile streaming due to significantly different usage scenarios. Furthermore, the consumers do not have a clear vision how much and what for they are willing to pay for. These conditions make it almost impossible to objectively perform a classical WTP survey in order to define the maximum amount of money that may be contributed by an individual to equalize the utility change. Therefore, we investigated the more fundamental WTP features. We define WTP as readiness to pay for provided quality of video streaming.

The paper is organized as follows: in Section 2, our video streaming usage scenario is described as well as the setup of our survey we performed to obtain MOS and WTP values. In Section 3, the mutual dependences between MOS and WTP will be explained. Section 5 contains conclusions and some final remarks.

\section{THE TEST SETUP FOR VIDEO QUALITY AND WTP EVALUATION}

For the tests we selected three sets of five video sequences each having a ten-second duration. Two sets were encoded in SIF resolution and one set in QCIF resolution. All sequences in SIF resolution were encoded with the H.264/AVC baseline profile $1 \mathrm{~b}$ and sequences in QCIF resolution were encoded with H.263 profile 3 and level 10. We choose the five most frequent contents for each resolution with different impact on the user perception: news, soccer, panorama for weather forecast, video clip, foreman (only with QCIF), cartoon (only with SIF), video clip (only with SIF), traffic (only with QCIF). 
For our survey the following frame rates (FR) were selected: 5fps; 7,5fps; 10fps; 15fps. The sequences encoded in SIF resolution were encoded on bit rates (BR) $24 \mathrm{kbit} / \mathrm{s}, 50 \mathrm{kbit} / \mathrm{s}$, $56 \mathrm{kbit} / \mathrm{s}, 60 \mathrm{kbit} / \mathrm{s}, 70 \mathrm{kbit} / \mathrm{s}, 80 \mathrm{kbit} / \mathrm{s}, 105 \mathrm{kbit} / \mathrm{s}$ and for QCIF resolution on following BRs: $18 \mathrm{kbit} / \mathrm{s}, 44 \mathrm{kbit} / \mathrm{s}, 80 \mathrm{kbit} / \mathrm{s}$. In total they were 36 combinations for SIF scenario and 54 combinations for QCIF scenario but we excluded some combinations where the resulting video quality was clearly insufficient.

To obtain MOS and WTP values, we worked with 74 test persons (36 persons with SIF resolution and 38 persons with QCIF resolution). The chosen group of test persons ranged different ages (between 20 and 30), gender, education and experience with image processing. The test method was absolute category rating (ACR) [10] as it better imitates real world streaming scenarios. We performed our tests on UMTS mobile terminals in this point we did not follow ITU-T Recommendation [10]. Three runs for QCIF and two runs for CIF of each test were taken. In order to avoid a learning effect we made a break of half an hour between the runs. Thus, the subjects did not have the original sequence as a reference, resulting in an enlarged variance. The test persons evaluated the video quality after each sequence using a five grade MOS scale (1-bad, ..., 5-excellent) in a prepared form. The WTP was evaluated with simple binary question:"If you should pay for this video sequence. Would you be satisfied with the video quality?" The question was designed in order to define a relation between WTP (as we define) and subjective quality of video streaming.

\section{RELATION BETWEEN WTP AND VIDEO QUALITY}

The Figures 1, 2 clearly show mutual linear dependence between MOS and WTP. Moreover, we can see that the behaviour is not dependant on the codec or the resolution.

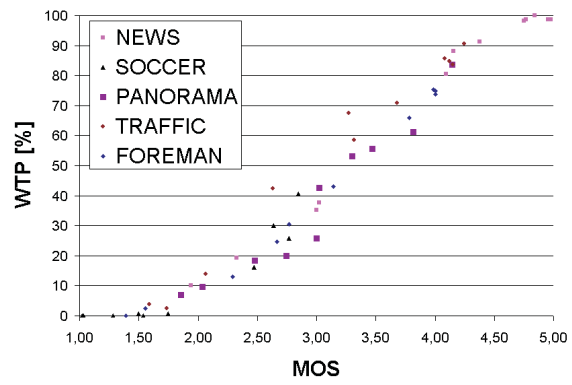

Fig. 1. Relation between MOS and WTP for QCIF resolution

These features allow us to estimate WTP with subjective video quality. Finally, we propose one universal metric for the mobile scenario which returns percentage of customers that are ready to pay for provided video quality:

$$
\mathrm{WTP}=31.514 \cdot \mathrm{MOS}-45.664 \text {. }
$$

For these two test cases the proposed metric is independent from codec, resolution and content. To validate the performance of our proposed metric, we used the Pearson (linear) correlation factor. In order to provide a detailed overview

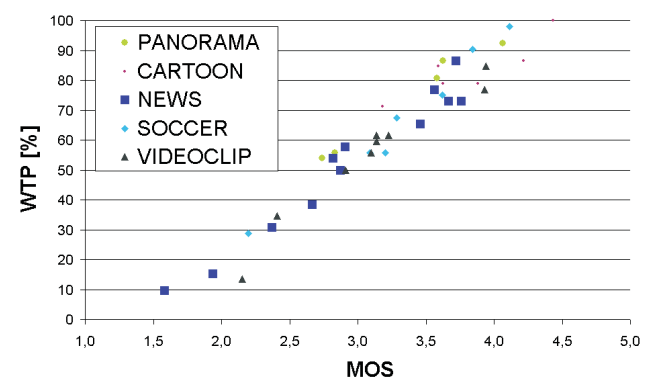

Fig. 2. Relation between MOS and WTP for SIF resolution

we calculated the Pearson correlation factor separately for both resolutions. Performance of proposed metric for QCIF resolution is $97.53 \%$ and for SIF $96.83 \%$. Finaly for both test cases was achieved $95.73 \%$ corelation.

\section{CONCLUSiOnS}

In this summary we present results from extensive survey for the most frequent mobile streaming scenarios. Furthermore, we show and describe strong mutual relation between WTP and subjective video quality (MOS). We proposed an universal WTP metric for mobile video streaming services and investigated its performance.

\section{ACKNOWLEDGMENT}

The authors would like to thank mobilkom austria AG for supporting their research. The views expressed in this paper are those of the authors and do not necessarily reflect the views within mobilkom austria AG.

\section{REFERENCES}

[1] O. Nemethova, M. Ries, A. Dantcheva, S. Fikar, M. Rupp: "Test Equipment of Time-Variant Subjective Perceptual Video Quality in Mobile Terminals," In IASTED International Conference on Human Computer Interaction, pp.14-16, Phoenix, USA, Nov. 2005.

[2] 3GPP TS 26.234 V6.8.0:'Transparent end-to-end Packet-switched Streaming Service (PSS); Protocols and codecs".

[3] ITU-T Recommendation H.264 (03/05): "Advanced video coding for generic audiovisual services" ISO/IEC 14496-10:2005: "Information technology - Coding of audio-visual objects - Part 10: Advanced Video Coding".

[4] J. Gozdecki, A. Jajszczyk, R. Stankiewicz: "Quality of service terminology in IP networks," IEEE Communications Magazine, pp.153-159, Mar. 2003.

[5] A. Watson, M. A. Sasse: "Measuring Perceived Quality of Speech and Video in Multimedia Conferencing Applications," In ACM Multimedia, pp.55-60, ACM, Oct. 1998.

[6] M. Ries, O. Nemethova, M. Rupp, "Motion Based Reference-Free Quality Estimation for H.264/AVC Video Streaming," Proc. of the ISWPC'07, San Juan, PR, USA, Feb. 2007.

[7] M. Ries, C. Crespi, O. Nemethova, M. Rupp, "Content Based Video Quality Estimation for H.264/AVC Video Streaming," Proc. of the IEEE WCNC'07, Hong Kong, Mar. 2007.

[8] Adima Manoli: "User Assessment for Negotiating the Quality of Service for Streaming Media Applications," Proc. of the 19th International Symposium on Human Factors in Telecommunication, Berlin, Germany, Dec. 2003.

[9] K.Yamori, H.Ito, Y.Tanaka: "Optimum Pricing Methods for Multiple Guaranteed Bandwidth Service," Proc. of the 2005 Networking and Electronic Commerce Research Conference, Riva Del Garda, Italy, pp.349355 , Oct. 2005

[10] ITU-T Recommendation P.910, "Subjective video quality assessment methods for multimedia applications," Sep. 1999. 